\title{
Persistencia y costo ambiental sostenible relativo ante arsénico total en sedimentos del río Chacapalca, provincia Lampa - Región Puno
}

\author{
Persistence and sustainable environmental cost related to total arsenic \\ in sediments of the Chacapalca river, Lampa Province - Puno Region \\ Recibido: junio 12 de 2018 | Revisado: julio 20 de 2018 | Aceptado: agosto 18 de 2018
}

\author{
MiRIAN VÁSQUEZ \\ George Argota Pérez ${ }^{2}$
}

1 Municipalidad Distrital de Ocuviri. Provincia Lampa. Puno, Perú mirianv.blga@gmail.com

2 Centro de Investigaciones Avanzadas y Formación Superior en Educación, Salud y Medio Ambiente "AMTAWI". Puno, Perú george.argota@gmail.com

\begin{abstract}
RESUMEN
El objetivo del estudio fue evaluar la concentración de arsénico total y el costo ambiental sostenible relativo en sedimentos del río Chacapalca de la provincia de Lampa, Región Puno. Entre septiembre y diciembre del 2017 se realizó un muestreo probabilístico aleatorio en tres puntos de exposición donde la cuantificación se realizó por espectrometría de absorción atómica con plasma, inductivamente, acoplado mediante digestión ácida. Con los valores hallados de arsénico total se calculó el costo ambiental sostenible relativo (por sus siglas en inglés, RESCO) el cual relacionó mediante un cociente, el costo de evaluación medido contra el costo preventivo normativo. Se encontró que las concentraciones de arsénico $(\mathrm{mg} / \mathrm{Kg})$ en los puntos de muestreo fueron: $8,39 \pm 0,01$ (1); 9,41 $\pm 0,01$ (2) y 6,33 $\pm 0,01$ (3) las cuales superaron el límite permisible (ISQG) según refirió la norma canadiense utilizada (CEQG: 5,9). Entre los valores hallados se observó que, existió diferencias estadísticamente significativas $(\mathrm{p}<0,05)$ entre los puntos de muestreo. Según el análisis del RESCO se obtuvo un cociente de 0,0 por lo que fue categorizado de forma integrada los sedimentos como recursos no sostenibles. Se concluyó que, ante las concentraciones elevadas de arsénico los sedimentos, existió contaminación ambiental lo cual, fue perjudicial como valoración sobre el estado de equilibrio del río Chacapalca. Asimismo, la calidad de los sedimentos no fue aceptada y al categorizarse como recurso no sostenible relativo, el valor de uso del río Chacapalca se indicó en condiciones limitadas.
\end{abstract}

Palabras clave: persistencia, costo ambiental sostenible relativo, arsénico total, sedimentos superficiales, río Chacapalca

\begin{abstract}
The objective of the study was to evaluate the total arsenic concentration and the relative sustainable environmental cost in sediments of the Chacapalca River of the province of Lampa, Region Puno. Between September and December of 2017, a random probabilistic sampling was carried out in three points of exposure where the quantification was performed by using atomic absorption spectrometry with plasma, inductively, coupled by acid digestion. With the values of total arsenic found, the relative sustainable environmental cost (RESCO) was calculated, which related by means of a quotient, the cost of evaluation measured against the normative preventive cost. It was noticed that the concentrations of arsenic $(\mathrm{mg} / \mathrm{Kg})$ at the
\end{abstract}


sampling points were: $8.39 \pm 0.01(1) ; 9.41 \pm 0.01(2)$ and $6.33 \pm$ 0.01 (3) which exceeded the permissible limit (ISQG) according to the Canadian standard used (CEQG: 5,9). Among the values found it was observed that there were statistically significant differences $(\mathrm{p}<0.05)$ between the sampling points. According to the RESCO analysis, a quotient of 0.0 was obtained, which caused sediments to be categorized as unsustainable resources. It was concluded that, in the presence of high concentrations of arsenic in the sediments, there was environmental contamination, which was detrimental as an assessment of the equilibrium state of the Chacapalca River. Likewise, the quality of the sediments was not accepted and when categorized as a relative nonsustainable resource, the use value of the Chacapalca River was indicated under limited conditions.

Key words: persistence, relative sustainable environmental cost, total arsenic, surface sediments, Chacapalca River

\section{Introducción}

El agua es un recurso necesario en cualquier parte del mundo (Elgallal, Fletcher \& Evans, 2016); y actualmente, está en desequilibrio como matriz ambiental dentro de los ecosistemas acuáticos, ya que las plantas de tratamientos convencionales no están diseńadas para la remoción de los contaminantes emergentes (Pal et al., 2014) y donde se conoce sus efectos endocrinos sobre la salud humana (Rodríguez et al., 2017), así como, numerosos procedimientos tecnológicos implementados en las plantas de tratamiento por lo general, tampoco eliminan la toxicidad de los metales pesados: MP (Lee \& Dhar, 2012; Dixit et al., 2015), lo cual hace que los efluentes sean vertidos con elevada peligrosidad, lo que resulta que su seguridad por valor de uso posterior sea poco eficiente.

Particularmente, la biodegradabilidad de los MP (Raval, Shah \& Shah, 2016) hace que puedan bioacumularse en organismos inferiores y luego, biomagnificarse (Molina, Ibañez \& Gibon, 2012) causando riesgos irreparables en la salud huma- na (Wang et al., 2015; Han et al., 2016; Londoño, Londoño \& Muñoz, 2016).

Uno de los elementos más tóxicos, ambientalmente, es el arsénico ya que las especies de este elemento se encuentran, principalmente, en las formas de arseniato $[$ As $(\mathrm{V})]$ y arsenito [As (III)] en aguas naturales y sedimentos (Gorny et al., 2015a). La solubilidad, movilidad y la toxicidad del arsénico depende de su estado de oxidación (Jain \& Ali, 2000; Dixit \& Hering, 2003). La determinación de la especiación As y la transformación, resulta esencial para comprender el comportamiento de As anóxicos en los sedimentos de cualquier río aunque, resulta de elevada complejidad debido a, las numerosas reacciones biogeoquímicas. Transformaciones de estas especies principales, combinadas con otros procesos incluso bacterianos son capaces de modificar profundamente la especiación y el destino de arsénico dentro de los primeros centímetros de la columna sedimentaria (reconocida como la huella histórica), así como su toxicidad potencial hacia los organismos acuáticos (Borch et al., 2009; Gorny et al., 2015b). 
Por otra parte, la predicción del riesgo ecotoxicológico por exposición a metales pesados ha sido medida mediante modelos informatizados (Chapman \& Riddle, 2003; Patlewicz \& Fitzpatrick, 2016), uso de índices (López, Figueroa \& Corrales, 2016) y por bioensayos (Fahd et al., 2014; Sadeghi \& Hedayati, 2014). Sin embargo, estas mediciones de forma independiente, quizás limiten algunas interpretaciones sobre la calidad ambiental económica y sostenible de los recursos acuáticos. La medición económica y sostenible sobre la calidad ambiental ha sido abordada desde diferentes perspectivas, externalidades, contingencias, derecho de propiedad, eficiencia económica y desde la razón sobre la pérdida del bienestar (Butler, Corvalan \& Koren, 2005).

Algunos enfoques intentan, además, establecer conexiones teóricas entre los sistemas ecológicos y económicos (Jia- jun et al., 2014), pero estas conexiones resultan muy complejas; posiblemente por la inexistencia valorativa en muchas legislaciones internacionales con razón a la orientación económica (David et al., 2004; Pérez, Peña \& Alvarez, 2011). El objetivo del estudio fue evaluar la persistencia y costo ambiental sostenible relativo ante arsénico total en sedimentos del río Chacapalca, provincia Lampa-Región Puno.

\section{Materiales y métodos}

El estudio se realizó en el río Chacapalca perteneciente a la Municipalidad Distrital de Ocuviri, provincia de Lampa, Región de Puno-Perú. Fueron seleccionados los sedimentos superficiales correspondientes a tres puntos de muestreo. Este río se extiende por una longitud aproximada de 15-18 Km. (Figura 1), aunque no existen informes técnicos que la justifique.

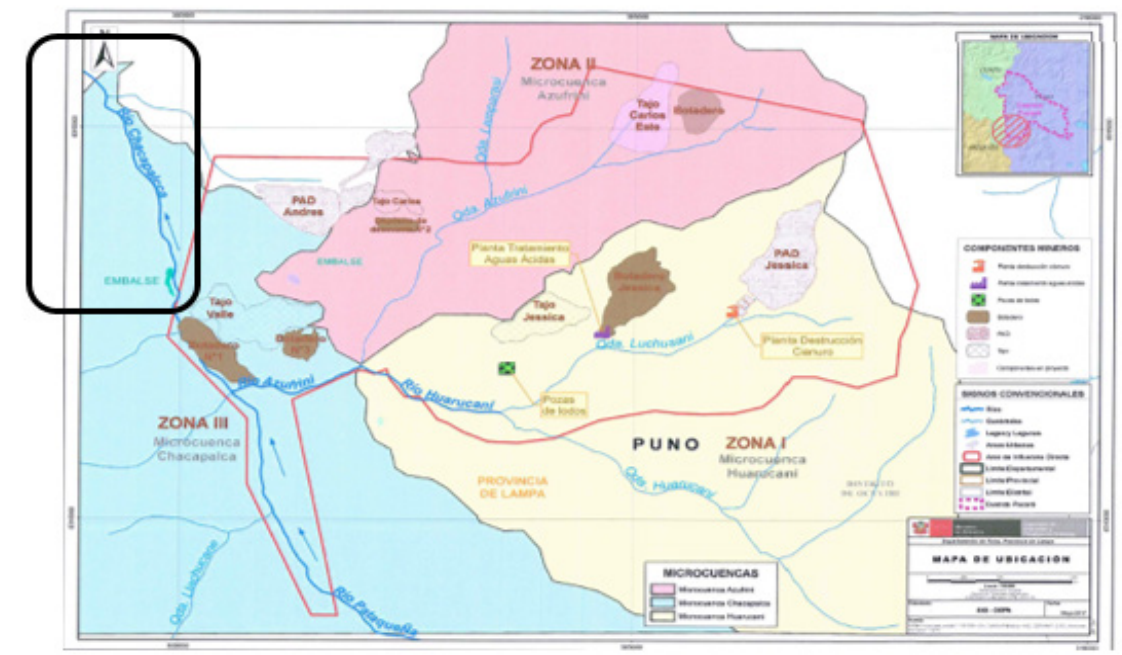

Figura 1. Mapa cartográfico del río Chacapalca (rectángulo) Fuente: Informe Técnico 054-2017-OEFA/DE-SDLB-CEAME

El análisis de los sedimentos correspondió a 5,0 cm de la capa de espesor superficial (Krueger, 1991), teniendo precaución de, no alterar la interfase agua- sedimento y solo generar resultados que corresponda a la matriz de interés (Gómez et al., 2000). 
Se seleccionó 1,0 Kg final de sedimentos depositándose en bandeja plástica durante 72 horas para su secado a temperatura ambiente. Luego se pesó 2,0 g del sedimento que fueron colocados en vaso de precipitado $(250 \mathrm{ml})$ y se ańadieron 40,0 ml de agua regia $\mathrm{HCl}: \mathrm{HNO}_{3}(3: 1)$ y calentó a temperatura de $180^{\circ} \mathrm{C}$ hasta obtener sales húmedas. Posteriormente, se ańadieron 5,0 $\mathrm{ml}$ de $\mathrm{HNO}_{3}(0,7 \mathrm{M})$ para disolver las sales húmedas y se enrasó con agua desionizada hasta un volumen 100,0 ml como lo señala Quevauvill (1993). La cuantificación del arsénico total se realizó por Espectrometría de Plasma Inductivamente Acoplado en el Laboratorio tipo Ambiental Acreditado ante la Dirección de Acreditación del Instituto Nacional de Calidad (INACAL-DA), además, del International Accreditation Service (IAS) bajo la Norma NTP ISO/IEC 17025 de Tipo Ambiental "Environmental Testing Laboratory S.A.C.

Debido a la inexistencia sobre determinada expresión matemática que refiera el posible costo ambiental sostenible con relación al análisis de parámetros físicoquímicos de calidad ambiental se utilizó lo propuesto por Argota, Argota \& Iannacone (2016) quienes sugirieron la siguiente fórmula:

$$
\text { RESCO }=\Sigma_{i}^{\mathrm{n}} \mathrm{COA} / \Sigma_{i}{ }_{i}^{\mathrm{nCONP}}
$$

\section{Donde}

- RESCO = costo ambiental sostenible relativo

- $\mathrm{COA}=$ costo de evaluación (medición de cumplimiento sobre parámetros)

- CONP = costo de prevención normativo (número de parámetros medidos)

- i) observación inicial y n) observación final

Mediante la fórmula indicada puede conocerse la sostenibilidad del recurso de acuerdo al cumplimiento de parámetros (COA), obteniéndose luego, un cociente según tipo de categorías que se muestran en las Tablas 1 y 2.

Tabla 1

\section{Criterio de puntuación}

\begin{tabular}{l|c}
\hline \multicolumn{1}{c|}{ Criterio } & PUnTUACIón \\
\hline Cumple el valor establecido por la norma regulatoria utilizada & 1 \\
No cumple el valor establecido por la norma regulatoria utilizada & 0 \\
\hline
\end{tabular}

\section{Tabla 2}

Categorias del costo ambiental sostenible relativo/intervalo

\begin{tabular}{|l|c|}
\hline \multicolumn{1}{|c|}{ Categoría de sostenibilidad relativa } & INTERVALO \\
\hline Recurso sostenible relativo & 1,0 \\
Recurso moderadamente sostenible relativo & $0,85-0,99$ \\
Recurso ligeramente sostenible relativo & $0,6-0,84$ \\
Recurso poco sostenible relativo & $0,41-0,59$ \\
Recurso no sostenible relativo & $0,0-0,4$ \\
\hline
\end{tabular}


Para el tratamiento de los datos se utilizó el programa estadístico Statgraphics Centurion XVI-II. La prueba de normalidad se realizó mediante el Test de Shapiro-Wilk que se basa en la comparación de los cuartiles de la distribución normal ajustada a los datos. La comparación de las media se efectuó mediante el análisis de varianza con tres réplicas usando para la comparación múltiple de rangos el Test de Bonferroni. Se consideró significativos los resultados cuando $\mathrm{p}<0,05$.

\section{Resultados y Discusión}

La Tabla 3 muestra las concentracio- nes de arsénico total en los puntos de muestreo (PM) seleccionados (reportado por el Laboratorio Envirotest: Anexo A). Como en el Perú no existe una norma ambiental que regula las concentraciones de metales en sedimentos, los resultados se compararon con los valores de la Guía de Calidad Ambiental Canadiense (por sus siglas en inglés, Canadian Environmental Quality Guidelines) para sedimentos de cuerpos de agua continental (CEQG). El ISQG (por sus siglas en inglés, interim sediment quality guideline) representa el límite permisible del elemento (As) en los sedimentos.

Tabla 3

Concentración de As en sedimentos $(\mathrm{mg} / \mathrm{Kg}$ )

\begin{tabular}{c|c|c|c}
\hline Elemento & \multicolumn{3}{|c}{ Punto de muestreo } \\
\cline { 1 - 1 } As & 1 & 2 & 3 \\
\cline { 2 - 3 } & $8,39 \pm 0,01$ & $9,41 \pm 0,01$ & $6,33 \pm 0,01$ \\
\hline ISQG & \multicolumn{3}{|}{5,9} \\
\hline
\end{tabular}

Dada la tabla anterior, las concentraciones de As en los PM estuvieron por encima lo recomendado pudiendo afec- tar la calidad ambiental del río. La Tabla 4 muestra el análisis de varianza, además, de la comparación de homogeneidad.

Tabla 4

Categorias del costo ambiental sostenible relativo/intervalo

\begin{tabular}{c|c|c|c|c|c}
\hline FV & SV & Gl & CM & Razón-F & Valor-P \\
\hline Entre grupos & 14,7704 & 2 & 7,3852 & 73852,00 & 0,0000 \\
Intra grupos & 0,0006 & 6 & 0,0001 & & \\
Total (Corr.) & 14.771 & 8 & & &
\end{tabular}

Pruebas de Múltiple Rangos (Tests de Bonferroni)

\begin{tabular}{|c|c|c|c|}
\hline PM & Media & \multirow{2}{*}{\multicolumn{2}{|c|}{$\begin{array}{c}\text { Grupos Homogéneos } \\
\text { a }\end{array}$}} \\
\hline 3 & 6,33 & & \\
\hline 1 & 8,39 & \multicolumn{2}{|r|}{$\mathrm{b}$} \\
\hline 2 & 9,41 & \multicolumn{2}{|r|}{ c } \\
\hline \multicolumn{2}{|r|}{ Contraste } & Sig. & Diferencia \\
\hline \multicolumn{2}{|r|}{ PM 1 - PM 2} & $\mathrm{a}$ & -1.02 \\
\hline \multicolumn{2}{|r|}{ PM 1 - PM 3} & $\mathrm{~b}$ & 2.06 \\
\hline \multicolumn{2}{|r|}{ PM 2 - PM 3} & c & 3.08 \\
\hline
\end{tabular}


La razón-F que en este caso fue igual a 73852,0 resulta de ser el cociente entre el estimado entre grupos y el estimado dentro de grupos. Puesto que el valor-P de la prueba-F fue menor que 0,05 , existe una diferencia estadísticamente significativa entre las medias de As total en los tres puntos de muestreo un nivel del $95.0 \%$ de confianza. El análisis del Test de Bonferroni indicó que las tres medias de As total entre los puntos de muestreo fueron diferentes. Cuando las fuentes tributan sus efluentes con As, generalmente, los sedimentos presentes concentraciones elevadas (Rieuwerts et al., 2014).

En un estudio realizado en el río San Pedro en el Estado de Aguascalientes, México (Guzmán et al., 2011) se encontró que más del 50\% de los sedimentos muestreados presentaron concentraciones de arsénico por encima a lo permisible, oscilando sus valores entre $56,803 \mathrm{mg} \cdot \mathrm{Kg}^{-1}$ (periodo de seca) y $86,808 \mathrm{mg} \cdot \mathrm{Kg}^{-1}$ (periodo de lluvia). En este estudio, los resultados estuvieron por debajo comparativamente, aunque puede considerarse en ambos estudios como exposición contaminante.

En otro estudio ejecutado en una bahía se encontró que la máxima concentración total de As $\left(17,7 \mathrm{mg} \cdot \mathrm{kg}^{-1}\right)$ superó dos veces la concentración del "intervalo de efectos bajo" (ERL = Effects Range-Low $\left.=8,2 \mathrm{mg} \cdot \mathrm{kg}^{-1}\right)$. Se indicó que la acumulación se debió a vertimientos y manejos irregulares de productos identificados en fuentes antropogénicas cercanas (Quevedo et al, 2014).

En cambio, durante un estudio efectuado en el río Haina, Santo Domingo (República Dominicana) se indicó que de ocho estaciones de muestreo, solo en una las concentraciones de As estuvieron por encima. En todos esos casos los niveles registrados estaban por debajo del límite de detección del equipo de Absorción Atómica. Las concentraciones oscilaron entre 1,7 y 9,517 4 ppm (Contreras, Pérez, Mendoza. \& Gómez, 2004).

Es conocido que la cuenca hidrográfica del lago Titicaca es de tipo endorreica y donde convergen diversos afluentes que generan contaminación procedente de la actividad minera, fundamentalmente. En un reciente estudio sobre cuantificación de metales en sedimentos superficiales de la bahía interior se concluyó que, los sedimentos superficiales de la bahía interior de Puno, no representan riesgo por exposición a metales totales, ya que sus concentraciones se encontraron en el rango de los valores permisibles. En el caso particular del As, las concentraciones en todas las estaciones fueron similares $\left(0,0001 \mathrm{mg} . \mathrm{L}^{-1}\right)$ según lo reportado (Moreno et al, 2018).

Dada las concentraciones de As en los sedimentos se procedió a la sustitución de los valores por cada PM según la fórmula del costo ambiental sostenible relativo.

- $\operatorname{RESCO}(\mathrm{PM} 1+\mathrm{PM} 2+\mathrm{PM} 3)=0$ $+0+0 / 3$

- $\mathrm{RESCO}=0 / 3=0,0$

La Tabla 5 muestra la categoría de sostenibilidad relativa del sedimento ante las concentraciones de As. Como el cociente hallado en la fórmula presentó valor de $0,0$ (intervalo $0,0-0,4)$ entonces, correspondió a la categoría de recurso no sostenible relativo siendo de consideración para la salud ambiental del río. 


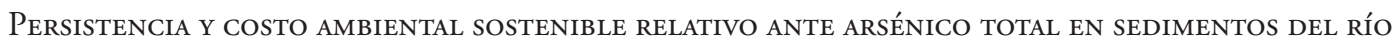
Chacapalca, provincia lampa - Región Puno

Tabla 5

Categorias del costo ambiental sostenible relativo/intervalo

\begin{tabular}{c|c|c}
\hline Categoría de sostenibilidad relativa & INTERVALO & RESULTADO \\
\hline & 1,0 & \\
& $0,85-0,99$ & \\
& $0,6-0,84$ & \\
& $0,41-0,59$ & \\
\hline Recurso no sostenible relativo & $0,0-0,4$ & 0,0 \\
\hline
\end{tabular}

De forma tradicional e histórica, los parámetros físico-químicos y microbiológicos de calidad del agua continua siendo lo realizado y quizás, rutinariamente, aunque siempre habrá que reconocer, el carácter temporal de sus interpretaciones durante un momento y espacio específico donde la limitación principal radica, en carecer de información referida con posibles efectos e impactos negativos sobre algunas poblaciones por exposición a contaminantes de interés (Argota, 2017).

La comparación de valores contra una referencia determinada representa una de las variantes por regulación ambiental ante posibles daños y en este sentido, al determinarse que las concentraciones de arsénico estuvieron por encima de lo permisible y ser el único elemento designado por interés, entonces se dictaminó como recurso no deseado. Sin embargo, uno de los actuales los problemas sociales y que sigue siendo contradictorio, es que los parámetros físico-químicos y microbiológicos, no dimensionan las respuestas ecotoxicológicas y por tanto, no arrojan total seguridad ambiental de modo tal que, otras herramientas requerían ser incorporadas. En este estudio, existió la limitación de analizar el comportamiento o respuesta sobre algún biomarcador con lo cual, quizás, podría precisarse o al menos correlacionar el costo estimado con la señal del biomarcador (Hamza, 2014; AbdAllah, 2017).

Dentro de las consecuencias sobre los protocolos de monitoreo ambiental es precisamente sus consideraciones por datos indicativos de parámetros, pero debe reconocerse que el ecosistema funciona como un todo entre factores abióticos y bióticos influenciados por el flujo de entrada y salida de elementos y donde solo uno de ellos, puede decidir la condición dinámica del recurso (Lock \& Bonventre, 2008; Argota \& Iannacone, 2017). En este sentido, la estimación del costo ambiental sostenible tiene su expresión en la relatividad debido a la disponibilidad de realizar un número determinado de mediciones que en cualquier otro caso o momento puede variar y aunque sea así, siempre podrá valorarse el recurso de la misma forma, pues la expresión matemática será la misma.

Unido a lo anterior, en términos de predicción de riesgo ambiental, las decisiones deben ser preocupantes aun cuando el contenido determinado sea como fracción total, pues al estar superando lo recomendable, entonces una parte podría presentar biodisponibilidad y ser finalmente dañino con lo que el recurso donde se encuentra el elemento no deseado, indicará del mismo modo, carácter no deseado para su uso. 


\section{Conclusiones}

1. Debido a las concentraciones elevadas de arsénico en los sedimentos con relación al límite permisible, se consideró que existió contaminación ambiental lo cual fue perjudicial como valoración sobre el estado de equilibrio del río Chacapalca.

2. La calidad de los sedimentos no fue aceptada y al categorizarse como recurso no sostenible relativo, el valor de uso del río Chacapalca se indicó en condiciones limitadas.

\section{Recomendaciones}

1. Se requiere realizar pruebas ecotoxicológicas sobre los sedimentos para señalar, posibles efectos no deseados (agudos y crónicos) en la biodiversidad local

2. Evaluar las concentraciones de otros metales, además, de parámetros físico-químicos para indicar grados de especiación química, persistencia ambiental y posibles efectos o respuestas de bioacumulación.

\section{Referencias}

AbdAllah, A.T. (2017). Efficiency of invertebrate animals for risk assessment and biomonitoring of hazardous contaminants in aquatic ecosystem, a review and status report. Environ Risk Assess Remediat; 1(1), 22-24. http://www.alliedacademies.org/environmental-risk-assessment-and-remediation/

Argota, P.G. \& Iannacone, O.J. (2017). Predicción cuantitativa mediante biomarcadores de uso permanente como nuevo criterio para biomonitores en ecotoxicología acuática. Revista The Biologist; 17(1), 141-153.

Argota, P.G. (2017). Monitoreo biológico ambiental en ecotoxicología acuática. Editorial Kopy Graft E.I.R.L., Cusco-Perú, 41, 10, 15, 16, 17. ISBN: 978-612-00-2558-1

Argota, P.G., Argota, C.H. \& Iannacone, O.J. (2016). Costo ambiental sostenible relativo a la variabilidad físico-química de las aguas sobre la disponibilidad de metales en el ecosistema San Juan, Santiago de Cuba-Cuba. The Biologist (Lima), 14(2), 219-232.

Borch, T., Kretzschmar, R., Kappler, A., Cappellen, P.V., Ginder-Vogel, M., Voegelin, A. \& et al. (2009). Biogeochemical redox processes and their impact on contaminant dynamics. Environ. Sci. Technol; 44, 15-23.

Butler, C.D., Corvalan, C.F. \& Koren, H.S. (2005). Human health, well-being, and global ecological scenarios. Ecosystems; 8(2), 153-162.

Chapman, P.M. \& Riddle, M.J. (2003). Missing and needed: polar marine ecotoxicology. Mar Pollut Bull; 46(8), 927-928. 
Contreras Pérez, J.B., Mendoza, C.L. \& Gómez, A. (2004). Determinación de metales pesados en aguas y sedimentos del Río Haina. Ciencia y Sociedad; 29(1), 38-71. http://www. redalyc.org/pdf/870/87029103. pdf

David, J., Múnera, O., Restrepo, F.C., Económica, V., Ambientales, D.E.C. \& Conceptual, M. (2004). Costos Ambientales: Marco Conceptual y Métodos. Semestre Económico, 7(13), 159-193.

Dixit, R., Wasiullah, Malaviya, D., Pandiyan, K., Singh, U. B., Sahu, A., et al. (2015). Bioremediation of heavy metals from soil and aquatic environment: An overview of principles and criteria of fundamental processes. Sustainability (Switzerland), 7(2), 2189-2212. https:// doi.org/10.3390/su7022189

Dixit, S. \& Hering, J.G. (2003). Comparison of arsenic (V) and arsenic (III) sorption onto iron oxide minerals: implications for arsenic mobility. Environ. Sci. Technol; 37, 4182-4189.

Elgallal, M., Fletcher, L. \& Evans, B. (2016). Assessment of potential risks associated with chemicals in wastewater used for irrigation in arid and semiarid zones: A review. Agric Water Manag; 177, 419-431.

Fahd, F., Khan, F., Hawboldt, K. \& Abbassi, R. (2014). Developing a novel methodology for ecological risk assessment of thiosalts. Stochastic Environmental Research and Risk Assemente; 28(2), 383-391.
Gómez, A.J.L., Giraldez, I., Sánchez, R.D. \& Morales, E. (2000). Selectivity assessment of a sequential extraction procedure for metal mobility characterisation using model phases. Talanta; 52, 545-554

Gorny, J., Billon, G., Lesven, L., Dumoulin, D., Madé, B. \& Noiriel, C. (2015b). Arsenic behavior in river sediments under redox gradient: a review. Sci. Total Environ; 505, 423-434.

Gorny, J., Dumoulin, D., Lesven, L., Noiriel, C., Madé, B. \& Billon, G. (2015a). Development and application of a HPIC-ICP-MS method for the redox arsenic speciation in river sediment pore waters. J. Anal. At. Spectrom; 30, 1562-1570

Guzmán, C.G., Ramírez, L.E.M., Thalasso, F., Rodríguez, N.S., Guerrero, B.A.L. \& Avelar, G.F.J. (2011). Evaluation of pollutants in water and sediments of the San Pedro river in the state of Aguascalientes. Universidad y Ciencia; 27(1), 1732. http://www.scielo.org.mx/pdf/ uc/v27n1/v27n1a2.pdf

Hamza, C.A. (2014). Usefulness of Bioindicators and Biomarkers in Pollution Biomonitoring. International Journal of Biotechnology for Wellness Industries; 3, 19-26.

Han, W., Fu, F., Cheng, Z., Tang, B. \& Wu, S. (2016). Studies on the optimum conditions using acidwashed zero-valent iron/aluminum mixtures in permeable reactive barriers for the removal of different heavy 
metal ions from wastewater. $J \mathrm{Ha}$ zard Mater; 302, 437-446.

Jain, C.K. \& Ali, I. (2000). Arsenic: occurrence, toxicity and speciation techniques. Water Res; 34, 43044312

Jiajun, H., Huimin, Z. Hui, Z., Xuan, G., Mingwei, S., Junhao, Z. \& Xiaotao, L. (2014). Ecological risk and economic loss estimation of heavy metals pollution in the Beijiang River. Ecol Chem Eng S; 21(2), 189-199.

Krueger, R.A. (1991). Focus groups: A practical guide for applied research. Beverly Hills; California: Sage

Lee, J. \& Dhar, B. (2012). Bio-processing of solid wastes and secondaLock, E.A. \& Bonventre, J.V. (2008). Biomarkers in translation; past, present and future. Toxicology; 245(3), 163-166. http://dx.doi.org/10.1016/j.tox.2007.12.004

Londoño, F.L.F., Londoño, M.P.T. \& Muñoz, G.F.G. (2016). Los riesgos de los metales pesados en la salud humana y Animal. Biotecnoloía en el sector Agropecuario y Agroindustrial, 14(2), 145. https://doi. org/10.18684/BSAA(14)145-153

López, I.D., Figueroa, A. \& Corrales, J.C. (2016). A Systematic Mapping of Water Quality Prediction Using Computational Intelligence Techniques. Revista Ingenierias Universidad de Medellin; 15(28), 35-51. http://dx.doi.org/10.22395/rium. v15n28a2
Molina, C.I., Ibañez, C. \& Gibon, F.M. (2012). Proceso de biomagnificación de metales pesados en un lago hiperhalino (Poopó, Oruro, Bolivia): Posible riesgo en la salud de consumidores Biomagnification process of heavy metals of a hiperhaline lake. Ecología en Bolivia; 47(2), 99-118.

Moreno, T.E., Argota, P.G., Alfaro, T.R., Aparicio, S.M., Atencio, L.S. Goyzueta, C.G. (2018). Cuantificación de metales en sedimentos superficiales de la bahía interior, lago Titicaca-Perú. Journal of High Andean Research; 20(1), 09-18. http://dx. doi.org/10.18271/ria.2018.326

Pal, A., He, Y., Jekel, M., Reinhard, M. \& Gin, K.Y. (2014). Emerging contaminants of public health significance as water quality indicator compounds in the urban water cycle. Environ. Int; 71, 46-62. http://dx.doi.org/10.1016/j.envint.2014.05.025

Patlewicz, G. \& Fitzpatrick, J.M. (2016). Current and future perspectives on the development, evaluation, and application of in silico approaches for predicting toxicity. Chem Res Toxicol; 29, 438-451. http:// dx.doi.org/10.1021/acs.chemrestox. 5 b00388

Pérez, M.A., Peña, M.R. \& Alvarez, P. (2011). Agro Industria Cañera y uso del agua: análisis crítico en el contexto de la política de agrocombustibles en Colombia. Ambiente \& Sociedade; XIV(2), 153-178. 
Quevauvill, P.H., Raurent, G. \& Griepink, B. (1993). Intern. J. Anal. Chem. 1993; 51, pp 231-235.

Quevedo, A.O., Villanueva, T.M., Barciela, A.Ma.C., Bermejo, B.P., Gómez, P.J. \& et al. (2014). Primer estudio de la contaminación por Arsénico en sedimentos de la bahía de Matanzas, Cuba. Revista Cenic Ciencias Quimicas; 45(1).

Raval, N.P., Shah, P.U. \& Shah, N.K. (2016). Adsorptive removal of nickel (II) ions from aqueous environment: A review. J Environ Manage; $179,1-20$

Rieuwerts, J.S., Mighanetara, K., Braungardt, C.B., Rollinson, G.K., Pirrie, D. \& Azizi, F., (2014). Geochemistry and mineralogy of arsenic in mine wastes and stream sediments in a historic metal mining area in the UK. Sci. Tot. Environ; 472,
$226-234$.

Rodríguez, O., Peralta, H.J.M., Goonetilleke, A. \& Bandala, R.E. (2017). Treatment Technologies for Emerging Contaminants in water: A review. Chemical Engineering Journal; $1-92$.

Sadeghi, A. \& Hedayati, A. (2014). Investigation of LC50, NOEC and LOEC of Glyphosate, Deltamethrin and Pretilachlor in Guppies (Poecilia reticulata). Iranian Journal of Toxicology; 8(26), 1124-1129.

Wang, Z., Zhang, X., Huang, Y. \& Wang, H. (2015). Comprehensive evaluation of pharmaceuticals and personal care products (PPCPs) in typical highly urbanized regions across China. Environ. Pollut; 204, 223232. http://dx.doi.org/10.1016/j. envpol.2015.04.021 
ANEXOS A. Reporte de los resultados / Envirotest
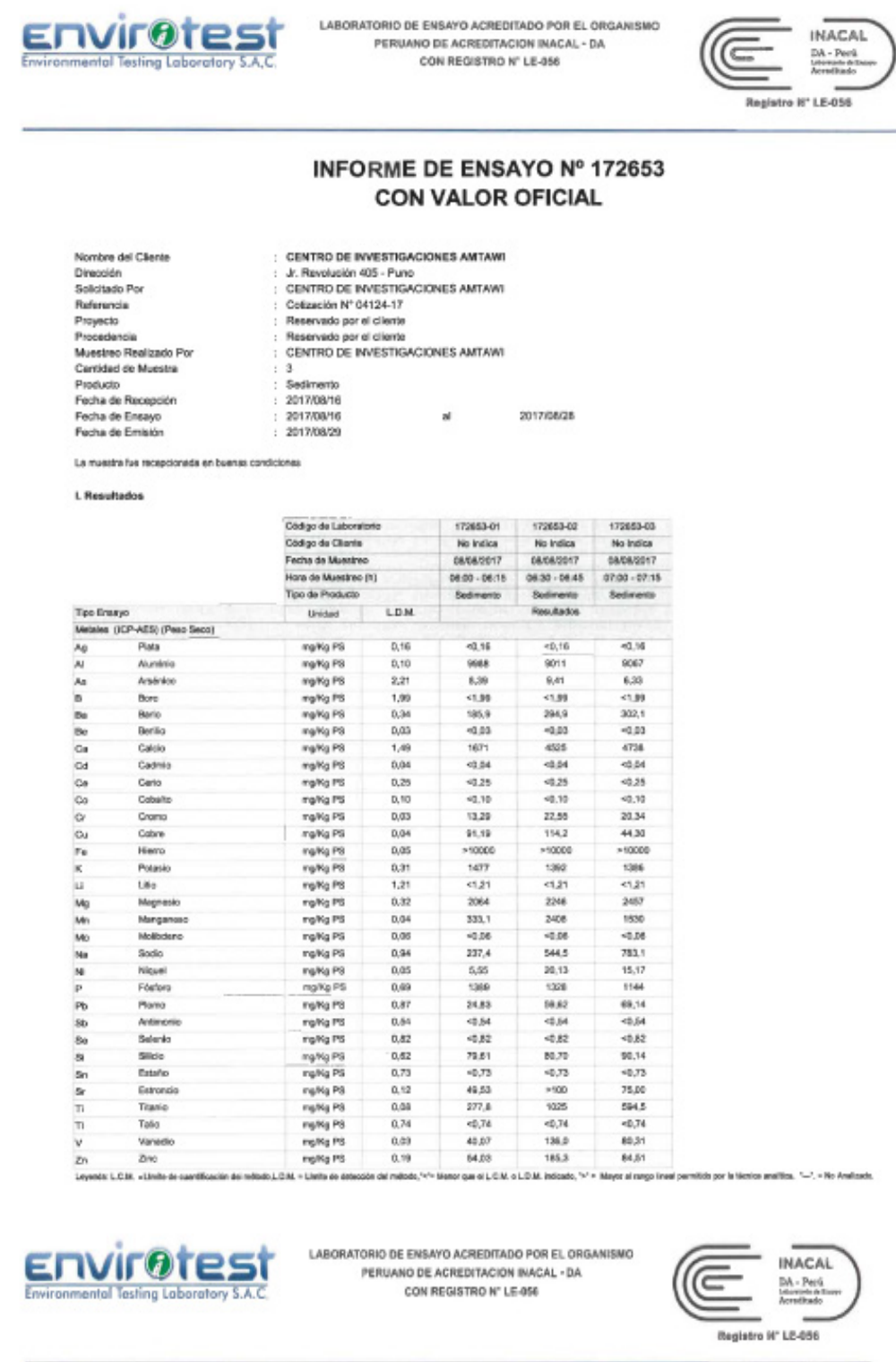

INFORME DE ENSAYO № 172653

CON VALOR OFICIAL

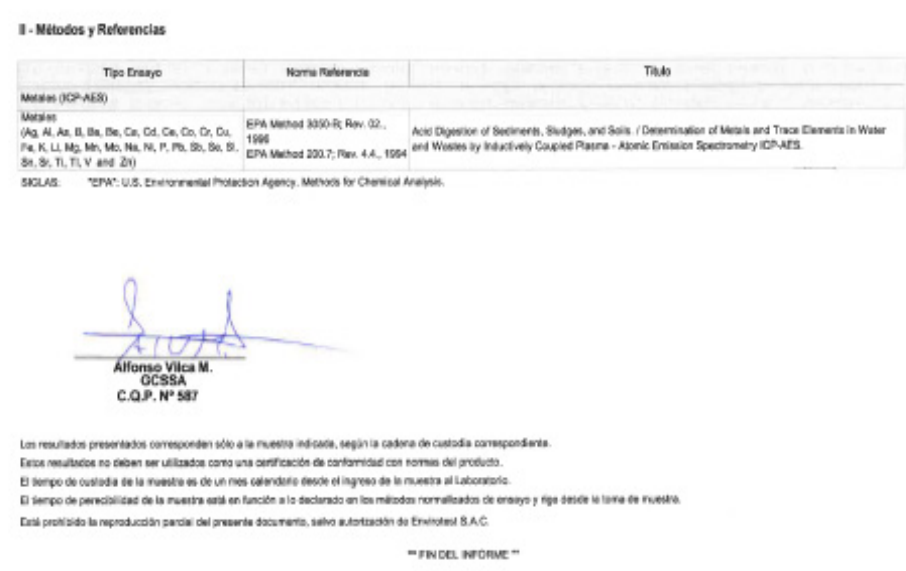

